\title{
Of light, of MEMS: Optical MEMS in telecommunications and beyond
}

\author{
F CHOLLET, H B LIU, M ASHRAF, B THUBTHIMTHONG, \\ $X$ M ZHANG, G HEGDE, A ASUNDI, V M MURUKESHAN and \\ A Q LIU
}

MicroMachines Centre, School of MAE, Nanyang Technological University, 50 Nanyang Avenue, 639798 Singapore

e-mail: franck.chollet@femto-st.fr

\begin{abstract}
The burst of the Internet bubble in 2000 has severely quenched the pace of development in the optical MEMS field. However, it is now clear that this field is again set to move forward as not only telecommunication but many other industries are benefiting from its application. We describe in this paper some of our latest achievements in the field, showing a new type of small scale optical switch based on integrated optics, a vibration/acceleration sensor using a veryshort external cavity laser and finally, the early development result of a nano-scale tunable photonic crystal that could be used for beam steering. The diversity of the applications we demonstrate, is a clear testimony that there is indeed 'plenty of room at the bottom' - and particularly in the natural combination of Light and MEMS!
\end{abstract}

Keywords. Optical MEMS; telecommunications; optical switch; external cavity laser sensor; tunable photonic crystal.

\section{Introduction}

The turn of the millennium saw an unprecedented effort put in MEMS R\&D when telecommunications venture capitalists understood that the technology could help solve the perceived bottleneck that the Internet was soon going to experience. Unfortunately the lack of market for products and services resulted in the burst of what was called the Internet bubble. Many companies disappeared (Onyx Microsystem, Xros, AIP Networks, Optical Micro Machines...) and it appeared for a while that optical MEMS would never recover.

However, optical MEMS go beyond telecommunications-and it is probably enough to be reminded of the highly successful DMD from Texas Instrument that powers the new digital movie theatres all over the globe and also scores of portable video projectors. Actually the application of MEMS to optics is very natural for a few reasons: for one, the wavelength of light, is in the $\mu \mathrm{m}$ range as the MEMS smallest features, then micro-forces (literally!) generated by micro-actuators have no difficulty in acting on massless photons (evidenced again by the micro-mirrors of the DMD) and finally, there are very few alternatives for the miniaturization of optical system. 


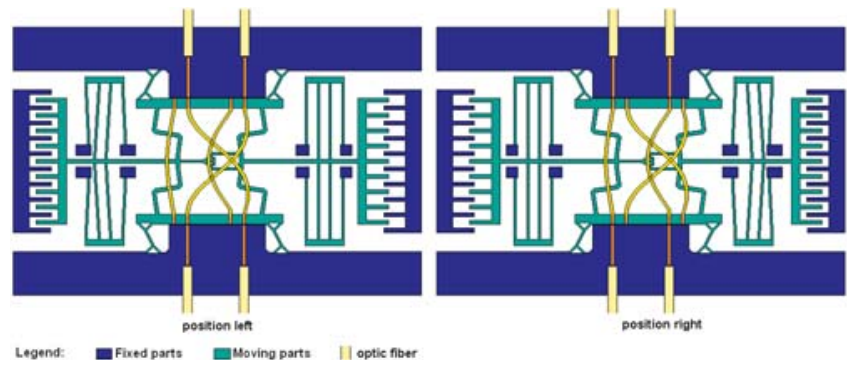

Figure 1. Principle of our Self-latching Waveguide Optical MEMS Switch (SWOMS).

In the following sections we will present a few devices that aim to show the large diversity of application of optical MEMS — of course, this work only encompasses a small fraction of what is possible, the list is really endless, bound only by our imagination.

\section{Optical switch with moving waveguide}

We recently developed a system that, still, belongs to the telecommunication application - a small scale $2 \times 2$ optical switch based on a mixture of integrated optics and MEMS actuator. The switch used as an aligned assembly of two structures produced separately: a wave-guiding network in polymer and a silicon actuator etched in a SOI wafer. The principle (Liu \& Chollet 2003) of the switch is shown in figure 1, where we see that depending on the position of the latching actuator different sets of waveguides connect the pairs of input and output fibres, yielding a true $2 \times 2$ functionality with one single switch element contrary to some earlier development in the field (Ollier et al 1995).

The device has many interesting features, as it is latching (it will not consume power during operation), able to minimise the gap between waveguides existing traditionally in similar devices (Ollier et al 1995) (decrease of the loss) and based on polymer waveguides (reduce the cost). Actually original aspects of the structure are also to be found in the detail of mechanical structure as we developed a new type of hinge dubbed the fork-hinge (Liu \& Chollet 2006a), in the waveguide network, that necessitated the use of advanced taper structure to maintain low loss in bend and for coupling with optical fibres, and in the fabrication process. Actually, owing to the large size of the actuator we had to develop a special process on SOI wafers, where we increased the gap beneath the structure by taking advantage of the lateral overtech appearing at the $\mathrm{Si} / \mathrm{SiO}_{2}$ interface. We found that this overetch could be controlled by following a series of simple rules to draw the layout of the system (Liu \& Chollet 2006b). Then the soft network of polymer waveguide is bonded to the actuator as shown in figure 2 . The tests that were conducted showed the relevance of the approach followed, even if it is clear that more development will be needed to obtain a commercial product (Liu \& Chollet 2007; Liu \& Chollet 2009). Figure 3 shows the NIR light from one of two input propagating in the bar and the cross state of the channel network toward each outputs.

\section{Vibration/acceleration sensor with very-short external cavity laser}

The use of external cavity laser for precise displacement measurement has a long history (Miles et al 1983; Lang \& Kobayashi 1980) using traditional technology. In such configuration (cf. figure 4) the intensity of the emitted light depends strongly on the phase of the light fed back 

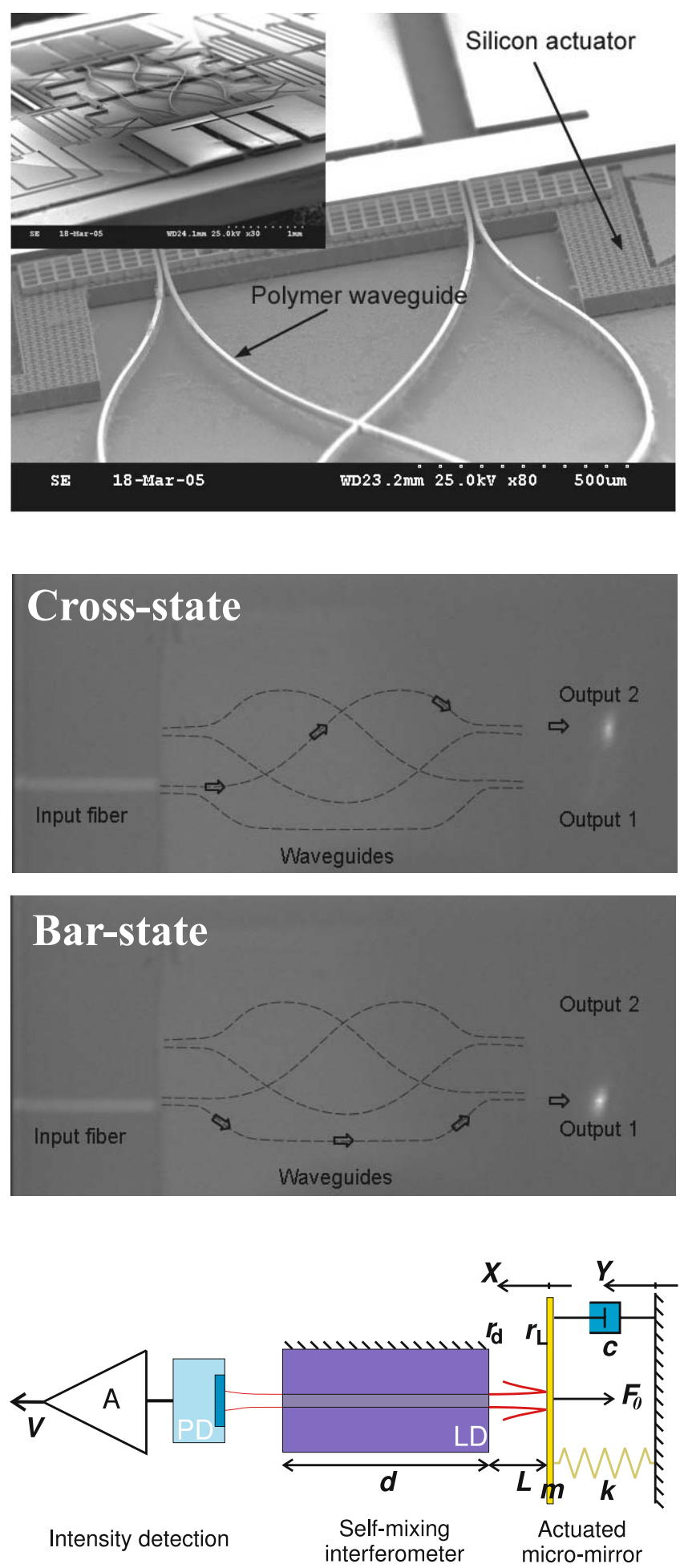

Figure 2. SEM view of finished SWOMS.

Figure 3. Test of SWOMS waveguides in NIR.

Figure 4. Principle of external cavity laser-based sensor. 


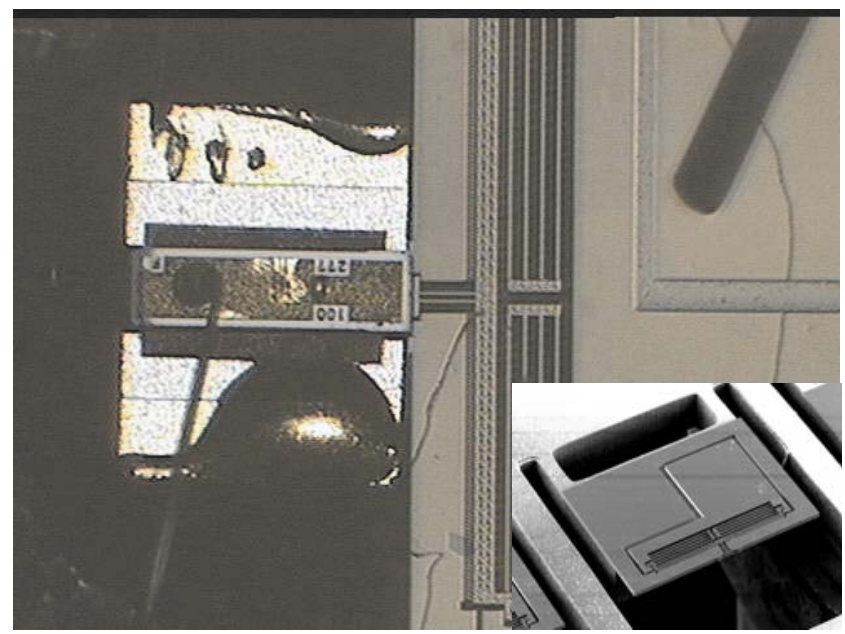

Figure 5. DRIE micromachined mirror packaged with diode laser and photodetector. The external cavity length is $10 \mu \mathrm{m}$.

in the cavity by the external mirror. If the mirror is suspended, its displacement is induced by acceleration or vibration of the mirror frame, allowing to measure it with very high accuracy. We have tried to propose a compact version of the system using a micromachined mirror in a very-short external cavity configuration (Chollet et al 2004). Actually, we showed that using an external cavity length below $10 \mu \mathrm{m}$ is increasing the packaging tolerance. Accordingly, we have designed two different versions of the micro-mirror with integrated actuator that allows fixing roughly the cavity length by using mechanical contact between bumps on the mirror and the laser chip, and fine tuning by applying a voltage to the actuator. The earlier version was based on surface micromachining technique (Chollet et al 2004), but more recently we used a DRIE etched mirror with improved manufactureability (cf. figure 5) that was packaged with a laser diode. Test of the external cavity system with different cavity length showed that the higher sensitivity obtained with the shorter cavity that could reach $5 \mathrm{pm} \sqrt{\mathrm{Hz}}$ at a vibration frequency of $2 \mathrm{kHz}$ as shown in the figure 6 . Thus, we were able to confirm the option chosen

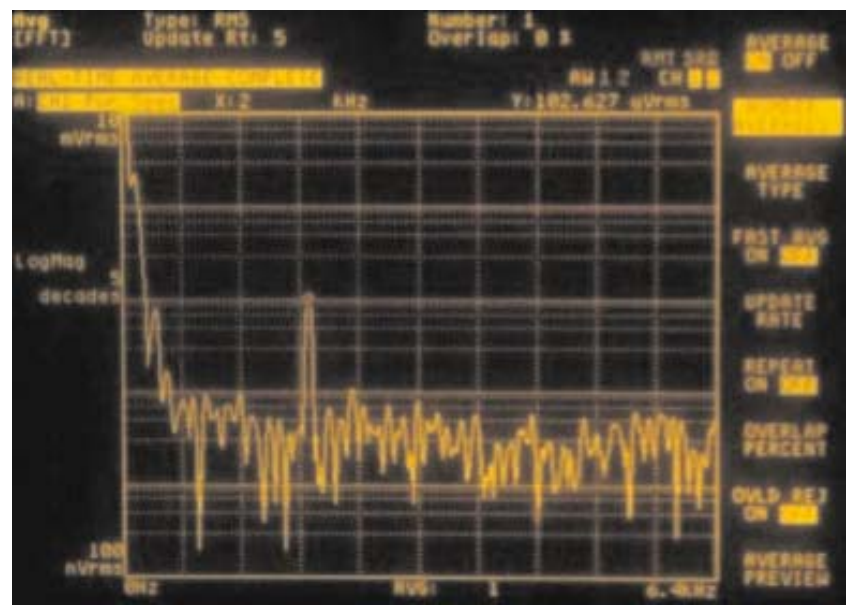

Figure 6. Spectrum of photodetector signal with an external mirror vibrated at $2 \mathrm{kHz}$ and an amplitude of $0.2 \mathrm{~nm}$. 


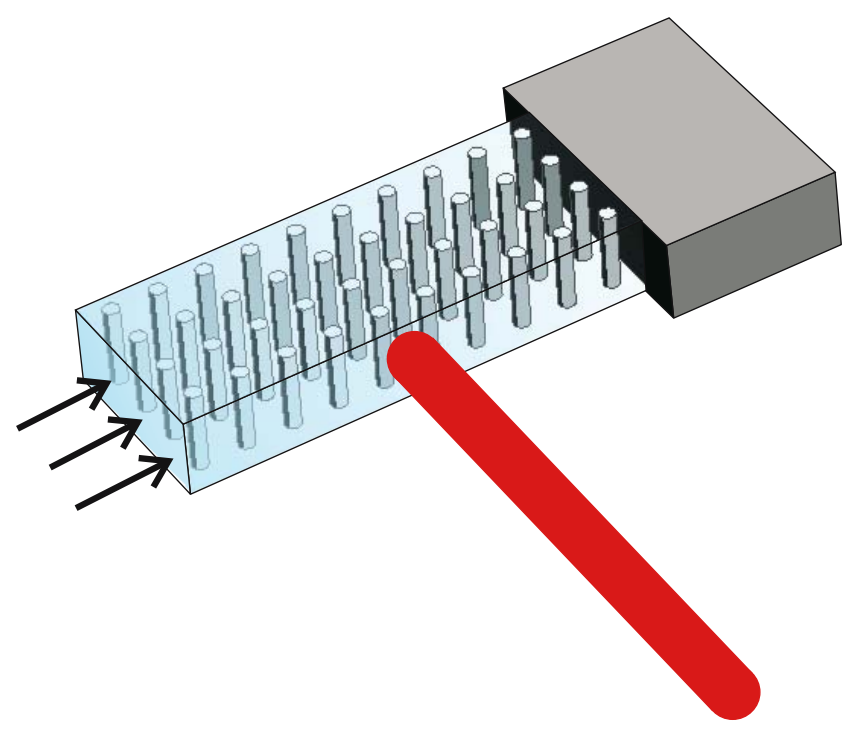

Figure 7. Mechanically tunable photonic crystal based on silicon micro-pillar in a transparent rubber matrix.

in the design, and current work focuses on optimising the structure and on the packaging. We are integrating the system on a silicon platform, using new technique for packaging of the hybrid system. This move should decrease the noise existing at low frequency as shown in figure 6 , allowing to use the system not only to measure vibration but also as a high sensitivity acceleration sensor.

\section{Tunable 2-D photonic crystal}

We recently proposed (Chollet 2004), simultaneously with a US team (Park \& Lee 2004), a new type of tunable photonic crystal based on mechanical tuning. The idea is to use an array of nano-pillars embedded in a polymer matrix and to deform the matrix by using

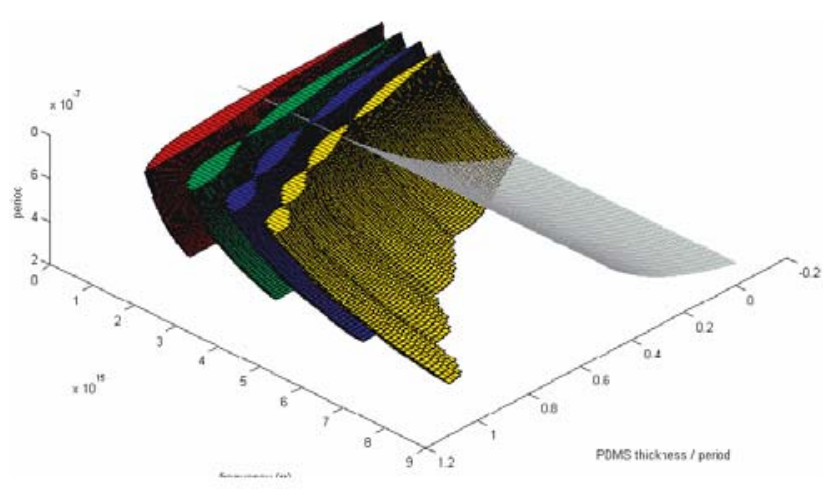

Figure 8. Position of the bandgap for a $1 \mathrm{D}$ tunable photonic crystal (Si/PDMS) as a function of the filling ratio between low index (PDMS) and high index (Si) zone and the compression of PDMS. 


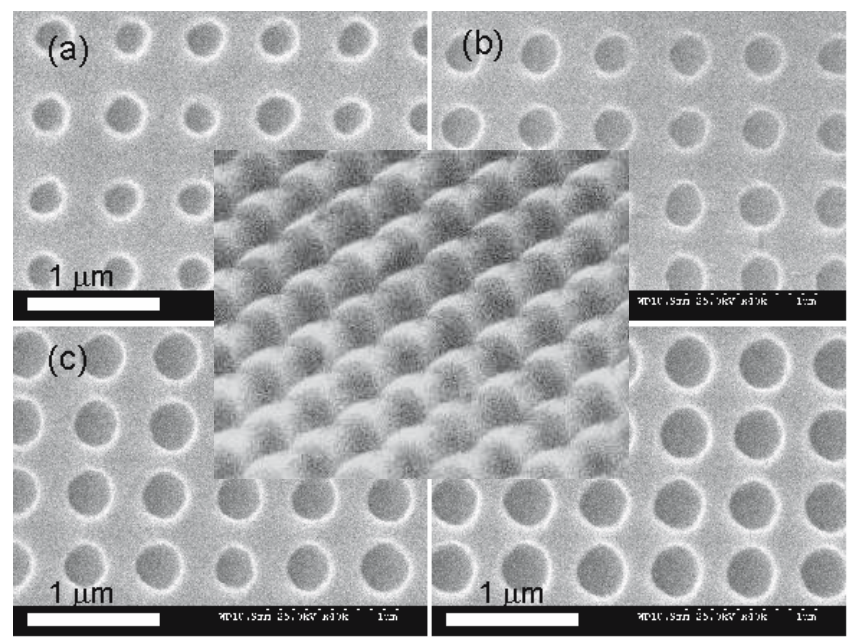

Figure 9. Effect (back) of exposure dose on e-beam lithography nano-pattern. Self (front) arrangement of $600 \mathrm{~nm}$ polystyrene spheres for shadow mask.

microactuator as shown in figure 7. The deformation of the array changes its period and the wavelength at which the photonic crystal is working, allowing interesting application, like beam steering by exploiting the superprism effect (Kosaka et al 1998; Lin et al 1996). Although other teams have proposed mechanical tunability of photonic crystal the unique use of pillars in a compliant matrix (instead of holes in a stiff crystal (Kim \& Gopalan 2001) has led to a potentially larger tuning range with more interesting capabilities. Actually the tuning of the structure needs first a thorough theoretical study, and its effect are actually difficult to represent simply. An example of the variation of the bandgap in a 1D photonic crystal based on the same principle is already complex as shown in figure 8 , where the compression of the structure results in an evolution along the greyed 'plane' that intersect the 3D structure of the bandgap (Thubthimthong \& Chollet 2008). The patterning of the photonic crystal has led to the development of cost effective nano-patterning in the sub-micrometre range where standard contact mask aligner can not work anymore figure 9. We have explored nano-imprinting,

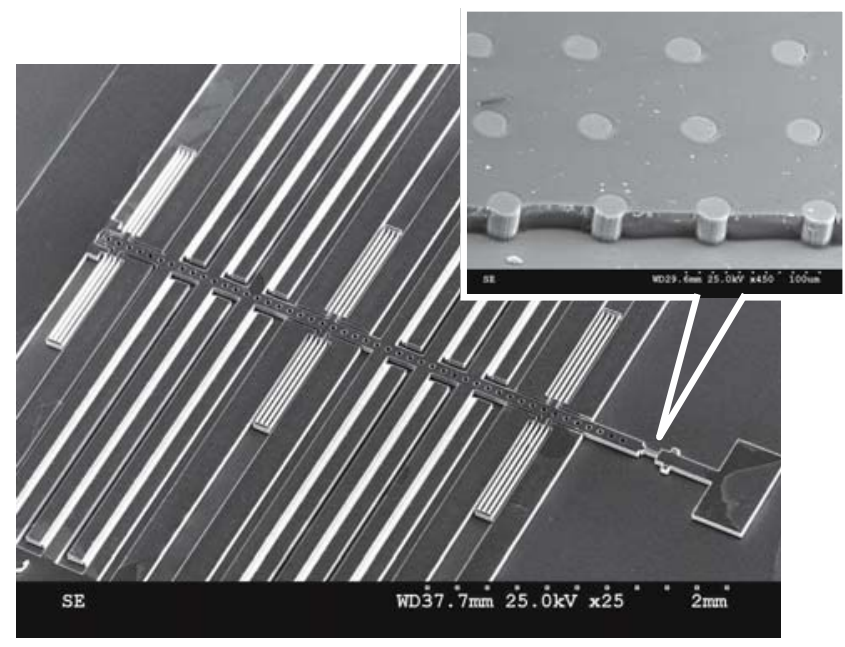

Figure 10. Large force micromachined electrostatic actuator and (inset) cross-section of scaled-up photonic crystal structure with $\mathrm{Si}$ pillars in a polymer matrix. 
e-beam (Cheng \& Chollet 2007) and self organisation of nano-particles (Ashraf et al 2007) to obtain different regular pattern for the operation of the photonic crystal in the $1.5 \mu \mathrm{m}$ telecommunication range.

Our latest achievement (cf. figure 10 are in the development of the actuator and in a process to produce the structure using CMP to precisely pattern the polymer matrix (Chaudhuri et al 2007). We hope in the coming year to be able to test our first tunable photonic crystal structure and present some of the applications we are currently pursuing.

\section{Conclusion}

The combined use of micro/nano-fabrication technique and optics has proved to be a fertile ground for developing new systems that were impractical to obtain only 10 years ago. If the pioneering work is already well advanced and some products have already moved to the industry - or have been proved to be failure — the field still appears to be boundless. For example, it easily goes to more fundamental directions by exploring nanometric scale and nanophotonics, or opening new applied research direction through the introduction of fluids, opening the whole new field of micro-opto-fluidics. Optics had for centuries been pushing technology that would allow to reach the sub-mircometric precision it needed, now that it has found it, it is our task to reach what our forefathers could only dream!

This work has been supported by the research grant RG8/02 (AcRF) from Nanyang Technological University, Singapore, and by a grant from A*STAR (Agency for Science, Technology and Research) SERC, Singapore.

\section{References}

Ashraf M, Sreenath A, Chollet F 2007 Low-cost mould for nano-imprinting uses monolayer of selforganized nanospheres. SPIE Newsroom, URL http://spie.org/x14253.xml?highlight=x2402

Chaudhuri B P, Thubtimthong B, Chollet F 2007 Development of fabrication process and electrostatic actuator for a mechanically tunable 2D photonic crystal. In International Conference on Materials for Advanced Technologies (ICMAT), July 2007, Singapore

Cheng W, Chollet F 2007 Effect of dose and pitch on size of e-beam patterned nano-dots. In International Conference on Materials for Advanced Technologies (ICMAT), July 2007, Singapore

Chollet F 2004 Development of 2D tunable photonic crystal for sensing and optical application. Technical report, A*STAR (Agency for Science, Technology and Research) SERC, Singapore

Chollet F, Hegde G M, Zhang X, Liu A, Asundi A 2004 Vibration measurement with a micromachined mirror in a very-short external cavity laser. Sensors and Actuators A, 116(2): 232-240

Kim S, Gopalan V 2001 Strain-tunable photonic bandgap crystals. Appl. Phys. Lett. 78: 3015

Kosaka H, Kawashima T, Tomita A, Notomi M, Tamamura T, Sato T, Kawakami S 1998 Superprism phenomena in photonic crystals. Phys. Rev. B58(16): R10096-R10099

Lang R, Kobayashi K 1980 External optical feedback effects on semiconductor injection laser properties. IEEE J. Quant. Electron. 16: 347-355

Lin S-Y, Hietala V M, Wang L, Jones E D 1996 Highly dispersive photonic band-gap prism. Optics Lett. 21(21): 1771-1773

Liu H, Chollet F 2003 Optical switch based on moving polymer waveguides and self-latching structure. Int. J. Computation. Eng. Sci. 4(3): 447-450 
Liu H, Chollet F 2006 Micro fork hinge for MEMS devices. J. Experimental Mech. 21(1): 61-70

Liu H, Chollet F 2006 Layout controlled one-step dry etch and release of MEMS using deep RIE on SOI wafer. IEEE/ASME J. MEMS 15(3): 541-547

Liu H, Chollet F 2007 Characterization of latching $2 \times 2$ optical switch with movable polymer waveguide. In Transducer's 07, Lyon, France

Liu H, Chollet F 2009 Moving polymer waveguides and latching actuator for $2 \times 2$ MEMS optical switch. IEEE/ASME J. MEMS 18(3): 715-724

Miles R, Dandridge A, Tveten A, Giallorenzi T 1983 An external cavity diode laser sensor. J. Lightw. Technol. 1: 81-93

Ollier E, Labeye P, Revol F 1995 Micro-opto mechanical switch integrated on silicon. Electron. Lett. 31(23): 2003-2005

Park W, Lee J-B 2004 Mechanically tunable photonic crystal structure. Appl. Phys. Lett. 85(21): $4845-4847$

Thubthimthong B, Chollet F 2008 Design of a 1D tunable photonic band gap filter. Microelectron. Eng. 85(5-6): 1421-1424 